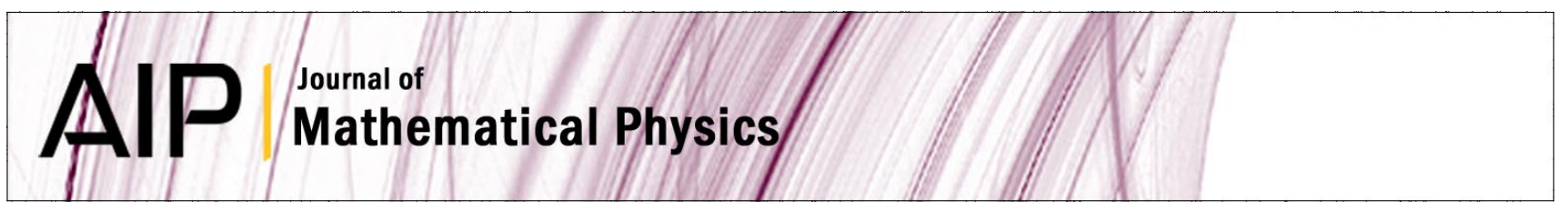

\title{
Variational principles for nonlinear dynamical systems
}

Vicenç Méndez

Citation: J. Math. Phys. 39, 954 (1998); doi: 10.1063/1.532363

View online: http://dx.doi.org/10.1063/1.532363

View Table of Contents: http://jmp.aip.org/resource/1/JMAPAQ/v39/i2

Published by the American Institute of Physics.

\section{Additional information on J. Math. Phys.}

Journal Homepage: http://jmp.aip.org/

Journal Information: http://jmp.aip.org/about/about_the_journal

Top downloads: http://jmp.aip.org/features/most_downloaded

Information for Authors: http://jmp.aip.org/authors

\section{ADVERTISEMENT}

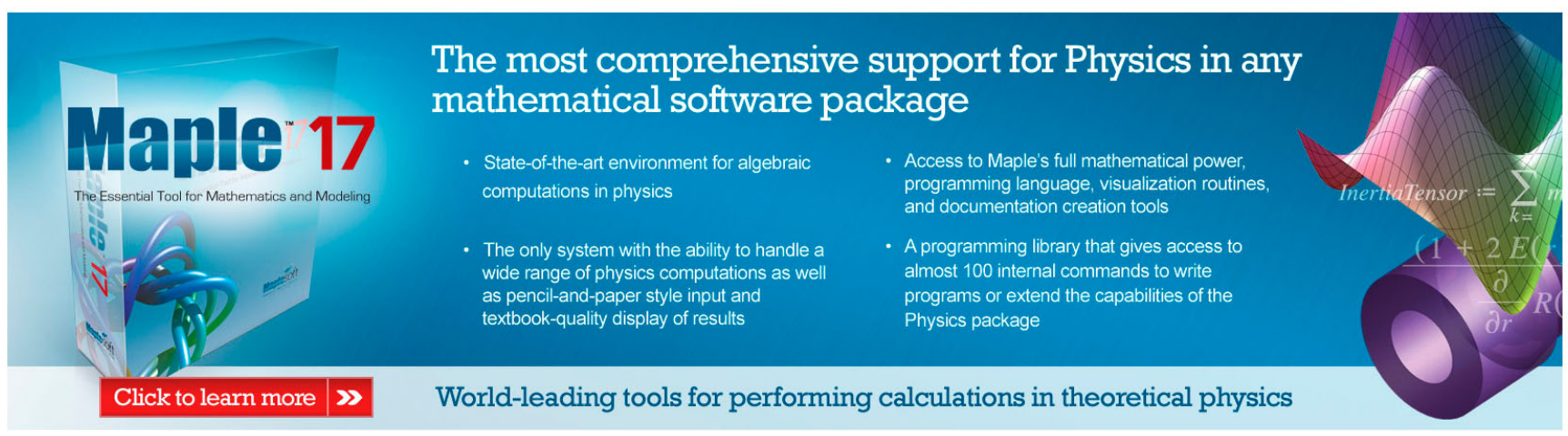




\title{
Variational principles for nonlinear dynamical systems
}

\author{
Vicenç Méndez \\ Grup de Física, Departament de Ciències Ambientals, Facultat de Ciències, \\ Universitat de Girona, C/. Albareda 3-5, 1701 Girona, Catalonia, Spain
}

(Received 26 February 1997; accepted for publication 8 September 1997)

A variational method for Hamiltonian systems is analyzed. Two different variational characterization for the frequency of nonlinear oscillations is also supplied for non-Hamiltonian systems. (C) 1998 American Institute of Physics.

[S0022-2488(98)02702-9]

\section{INTRODUCTION}

The dynamical study of nonlinear oscillatory systems is often reduced to numerical calculus or to some approximated analytical techniques. Most of them are based in perturbation methods ${ }^{1}$ as for instance the Poincaré-Lindsted method, the averaging (Krylov-Bogoliuvov-Mitropolsky) method, the Shohat expansion, or the multiscale method, to mention a few. They provide approximate solutions as well as the relationship between the frequency of the nonlinear oscillations and a small parameter (physically identified for the system, as the amplitude of the oscillations). Other approximate techniques consist in the qualitative analysis on the phase space by linearizing the differential equation around the fixed points. Periodic or exponential behavior of the system may be predicted after studying the stability of the fixed points.

Many different physical systems reduce to one-dimensional nonlinear ODE of second order, which may be studied by using the approximate techniques mentioned above. ${ }^{2,3}$ An important property of them is that the frequency depends explicitly on the amplitude of the oscillations. As we have mentioned, the perturbative techniques yield to an approximate solution in power series of the amplitude only.

Recently, Benguria and Depassier (BD for short $)^{4}$ have made use of a variational principle in order to solve the bifurcation problem for second-order nonlinear Hamiltonian systems. They also apply the principle to obtain simple approximate closed formulas for the frequency of largeamplitude oscillations.

In this work we extend this method to other situations. Basically, the method consists in transforming the differential equation $\ddot{u}+u=N(u)$, which may be written in the phase space variables $(p, u)$, with $p=-u^{\prime}$ (where the prime means differentiation with respect to the variable $\tau=2 \omega t / \pi)$ in an integral equation multiplying the differential equation by an auxiliary function $g(u)$, and integrating. The variational method provide a lower bound for an integral functional. From the Euler-Lagrange equations for the functional, one may calculate the auxiliary function $g(u)$ at a first approximation by taking $p(u)$ from the linear case $(N=0)$.

First of all, we generalize this transformation for Hamiltonian systems. We multiply the differential equation by $p^{n} g$ and apply the variational method. From this generalization we obtain the first interesting result: the variational characterization depends strongly on the exponent $n$. In this sense, we obtain a better fit for $n=-1$ than for $n=0$ (the BD's case).

However, as the systems are Hamiltonian, it is possible to find a relationship between $p$ and $u$ in the nonlinear situation. Using it, instead of its linear approach, one can find a new relationship for $g$ being the variational result for the frequency, always equal to the exact solution. This is shown in three specific and physically interesting cases.

Second, as non-Hamiltonian systems are very often found in practice and arise in many physical systems, we introduce in this work a special analysis for them. Following the same ideas employed for Hamiltonian systems, one obtains an explicit dependence of the variational characterization on $p(u)$. For non-Hamiltonian systems it is not possible to obtain a relationship between $p$ and $u$, at least exactly. In consequence, we adopt two ways to proceed. From one hand, we apply twice the variational method and find two coupled Euler-Lagrange equations, one of them corresponding to the same functional that appears in Hamiltonian systems and the other one to the 
new one that contains $p(u)$. The consequent variational characterization does not depend now on $p(u)$. This method also apply for large amplitude. On the other hand, we find for a class of non-Hamiltonian systems in which the above method does not hold, a perturbative solution to $p(u)$ for small nonlinearities. As a special example we treat the Van der Pol oscillator.

\section{VARIATIONAL PRINCIPLES FOR HAMILTONIAN SYSTEMS}

In this section we generalize the BD's method and we show, by using the Duffing oscillator as an example, the generality of the principle. Starting from a nonlinear Hamiltonian dynamical system of the form

$$
\ddot{u}+u=N(u), \quad \text { with } u(0)=a, \quad \dot{u}(0)=0,
$$

this becomes

$$
u^{\prime \prime}+\lambda u=N(u, \lambda)
$$

where the prime symbol stands for the differentiation respect to the angular variable $\tau=2 \omega t / \pi, \omega$ being the angular frequency of the nonlinear system (1) and $\lambda=(\pi / 2 \omega)^{2}$. We restrict our study to $u \in(0, a)$, where $a$ is the initial amplitude of movement, so $u(\tau=1)=0$. Defining the variable $p=-u^{\prime}>0$ we reduce the order of (2), and it is written as

$$
p \frac{d p}{d u}+\lambda u=N(u, \lambda) .
$$

Multiplying (3) by $p^{n} g(u)$, where $g(u)$ is an auxiliary function to be determined such that $g(0)=0$, and integrating, we obtain

$$
\lambda \int_{0}^{a} u p^{n} g(u) d u=\int_{0}^{a}\left(p^{n} N(u, \lambda) g(u)+\frac{1}{n+2} p^{n+2} g^{\prime}(u)\right) d u .
$$

Notice that for $n=0$ we recover the Benguria and Depassier case. We consider now, for a fixed $g$ and $n>-2$, the functional

$$
J_{g}[v]=\frac{(-1)^{n}}{n+2} \int_{0}^{a}\left(v^{\prime}\right)^{n+2} g^{\prime}(v) d v=\frac{(-1)^{n+1}}{n+2} \int_{0}^{1}\left(v^{\prime}\right)^{n+3} g^{\prime}(v) d \tau,
$$

defined for $v$ such that it satisfies $v(0)=a, v(1)=0$, and $v^{\prime}<0$ in $\tau \in(0,1)$. Defining $\phi\left(v, v^{\prime}\right)$ $=(-1)^{n+1}\left(v^{\prime}\right)^{n+3} g^{\prime}(v) /(n+2)$, the Euler-Lagrange equations for $J_{g}$ may be integrated once to obtain $\phi-v^{\prime} \partial \phi / \partial v^{\prime}=$ const, provided that $\phi$ does not depend explicitly on $\tau$. From the EulerLagrange equation we obtain

$$
(-1)^{n+1}\left(\tilde{v}^{\prime}\right)^{n+3} g^{\prime}(\tilde{v})=K>0,
$$

where $\widetilde{v}$ is the value of $v$ such that for a fixed $g, J_{g} \geqslant 0$ has a unique minimum. The auxiliary function $g$ may be obtained by integrating (6). So, once $g$ is determined, we get

$$
J_{g}[v] \geqslant J_{g}[\widetilde{v}]=\frac{K(a)}{n+2},
$$

where $K(a)$ is determined through the boundary condition on $\widetilde{v}$. So, we have from (4), (5), and (7),

$$
\lambda \geqslant \frac{\int_{0}^{a} N(u, \lambda) g(u) d u+\frac{K}{n+2}}{\int_{0}^{a} u p^{n} g(u) d u} .
$$


This constitutes the result of our first variational principle applied to nonlinear Hamiltonian oscillators. Notice the strong dependence of the variational principle on the exponent $n$, which we will show later in a specific example. For values of $n$ lower than -2 this variational principle does not apply and for the other values the upper bound for the frequency depends explicitly on $n$.

If we take $n=0$ in (8), we recover the Benguria and Depassier situation, ${ }^{4}$ where (8) reduces to

$$
\lambda \geqslant \frac{\int_{0}^{a} N(u, \lambda) g(u) d u+\frac{K}{2}}{\int_{0}^{a} u g(u) d u},
$$

and (6) is $\left(u^{\prime}\right)^{3} g^{\prime}(u)=-K$. Taking $n=-1$, (8) becomes

$$
\lambda \geqslant \frac{\int_{0}^{a} \frac{N(u, \lambda) g(u)}{p(u)} d u+K}{\int_{0}^{a} \frac{u g(u)}{p(u)} d u},
$$

and the Euler-Lagrange equation reduces to $\left(u^{\prime}\right)^{2} g^{\prime}(u)=K$.

Following Ref. 5, we can also derive, from another variational principle, a new restriction on the characteristic parameters of the system. Taking $n=0$ in (4), we may define the function

$$
\psi(p)=\lambda f(u) g+\frac{1}{2} p^{2} g^{\prime}(u),
$$

where $N(\lambda, u)=\lambda f(u)$. This function has, for each value of $u$, a minimum at $p_{\min }=0$, provided that $g^{\prime}(u)>0$. So $\psi\left(p_{\min }\right)=\lambda f(u) g \leqslant \psi(p)$, and finally

$$
\int_{0}^{a} u g(u) d u \geqslant \int_{0}^{a} f(u) g(u) d u .
$$

This constitutes the second variational principle for Hamiltonian systems. Let us now apply both principles on the Duffing oscillator and the nonlinear pendulum.

\section{A. The Duffing oscillator}

It is known that the exact solution for the Duffing oscillator, ${ }^{2}$

$$
\ddot{u}+u+\delta u^{3}=0, \quad \text { with } u(t=0)=a, \quad \dot{u}(t=0)=0,
$$

is given by

$$
u(t, \delta)=a \operatorname{cn}\left(t \sqrt{1+\delta a^{2}} ; k\right),
$$

where $\mathrm{cn}$ is the Jacobi elliptic function and $k^{2} \equiv \delta a^{2} / 2\left(1+\delta a^{2}\right)$. The exact expression for the frequency is then

$$
\omega_{\mathrm{ex}}=\frac{\pi}{2} \sqrt{1+\delta a^{2}} \frac{1}{F\left(\frac{\pi}{2}, k\right)},
$$

where $F(k)$ is called the complete elliptic integral of the first order. Taking $n=0$ in (8) we recover the BD situation, ${ }^{4}$ which yields

$$
\omega_{n=0} \leqslant \sqrt{1+\frac{3}{4} \delta a^{2}}
$$


Let us show in this example and in the following one the dependence of the frequency on the value of $n$ taken in (8). First, we analyze the linear case, for the Duffing oscillator, and compare the results obtained for $n=0$ and for $n=-1$. In this case, (10) leads to

$$
\lambda \geqslant \frac{K}{\int_{0}^{a} \frac{u g(u)}{p(u)} d u},
$$

$K$ and $g(u)$ must be calculated from

$$
\left(u^{\prime}\right)^{2} g^{\prime}(u)=K .
$$

In the linear case $(N=0)$, corresponding to the linear oscillator, one has $\left(u^{\prime}\right)^{2}=\lambda\left(a^{2}-u^{2}\right)$. Replacing this in (17), we obtain

$$
g(u)=\ln \left(\frac{a+u}{a-u}\right),
$$

as the expression for the auxiliary function. Solving (17) with $u(0)=a, u(1)=0$, one obtains $K=a \pi^{2} / 2$. On the other hand, defining $x \equiv u / a$, one has, from (18),

$$
\int_{0}^{a} \frac{u g(u)}{p(u)} d u=\frac{a}{\sqrt{\lambda}} \int_{0}^{1} \ln \left(\frac{1+x}{1-x}\right) \frac{x d x}{\sqrt{1-x^{2}}}=\frac{a \pi}{\sqrt{\lambda}} .
$$

Finally, from (16) we find $\lambda \geqslant(\pi / 2)^{2}$, which is the same result obtained by $\mathrm{BD} .^{4}$ For the nonlinear case $(N \neq 0)$, we find a different result, as we show in turn. The main difference between the cases $n=-1$ and $n=0$ is that, to solve the latter, one does not need the relationship between $p$ and $u$, while for $n=-1$ this is necessary. For the Duffing oscillator, we find, by making use of the Hamiltonian as a constant of motion, that

$$
p=\sqrt{\lambda a^{2}\left(1+\frac{\delta a^{2}}{2}\right)} \sqrt{\left(1-x^{2}\right)\left(s x^{2}+1\right)},
$$

where $s \equiv \delta a^{2} /\left(2+\delta a^{2}\right)$. From (10) and defining the following integrals:

$$
\begin{aligned}
& I_{1}(s)=\int_{0}^{1} \ln \left(\frac{1+x}{1-x}\right) \frac{x d x}{\sqrt{\left(1-x^{2}\right)\left(s x^{2}+1\right)}}, \\
& I_{1}(s)=\int_{0}^{1} \ln \left(\frac{1+x}{1-x}\right) \frac{x^{3} d x}{\sqrt{\left(1-x^{2}\right)\left(s x^{2}+1\right)}},
\end{aligned}
$$

we obtain

$$
\lambda \geqslant\left(\frac{\pi^{2}}{2}\right)\left(1+\frac{\delta a^{2}}{2}\right) \frac{1}{\left(I_{1}+\delta a^{2} I_{2}\right)^{2}} .
$$

The integrals $I_{1}$ and $I_{2}$ may be calculated, after some integrations by parts, in terms of the complete elliptic integral of the second kind, and we find finally for the frequency that

$$
\omega_{n=-1} \leqslant \frac{2}{\pi} \sqrt{1+\delta a^{2}} E\left(\frac{\pi}{2}, \sqrt{\frac{s}{s+1}}\right) .
$$

In Fig. 1 we represent the exact value $\omega_{\text {ex }}$ given by (14), the BD solution $\omega_{n=0}$ given by (15) in solid lines, and the new approach $\omega_{n=-1}$ given by (21) in dashed lines. As one can see, the 




FIG. 1. The frequency of the Duffing oscillator obtained by the BD method ( $\omega_{n=0}$, solid lines), the new variational method $\left(\omega_{n=-1}\right.$, dashed lines) versus the numerical exact solution $\left(\omega_{\mathrm{ex}}\right.$, solid line).

latter is a slightly better result than the BD solution. So, we have shown the dependence of the frequency on the value of $n$. Applying now the second variational method (11) to the Duffing equation, and taking the $\mathrm{BD}$ method we find the following restriction:

$$
\frac{-4}{3 a^{2}} \leqslant \delta .
$$

For $\delta>0$ the second variational method does not add any additional information, but for $\delta<0$ this may be understood ${ }^{3}$ as a bound for $\delta$, that is, $\delta \leqslant 4 / 3 a^{2}$. For $\delta<0$ we have that

$$
\omega_{n=0} \leqslant \sqrt{1-\frac{3}{4} \delta a^{2}} .
$$

The frequency has real value if $\delta \leqslant 4 / 3 a^{2}$, which coincides with the result obtained by using the second variational method.

\section{B. The nonlinear pendulum}

We study with some detail another very known nonlinear dynamical system namely the nonlinear pendulum. First of all we show that the $n=-1$ case may lead us to the exact solution. Later, we apply the BD approach to this solution. As it is widely known, the equation for the nonlinear pendulum is given by 


$$
\ddot{u}+\frac{g}{l} \sin (u)=0, \quad \text { with } u(t=0)=a, \quad \dot{u}(t=0)=0 .
$$

Defining again $\tau=2 \omega t / \pi$ and

$$
\lambda \equiv\left(\frac{\pi}{2}\right)^{2} \frac{g}{l}
$$

and taking into account the conditions $u(t=0)=a$ and $u(t=\pi / 2 \omega)=0,(22)$ is written as

$$
u^{\prime \prime}+\lambda \sin (u)=0, \quad \text { with } u(\tau=0)=a, \quad u^{\prime}(\tau=1)=0 .
$$

The exact solution ${ }^{2}$ is given by

$$
\omega_{\mathrm{ex}}=\frac{\pi}{2} \sqrt{\frac{g}{l}} \frac{1}{F\left(\frac{\pi}{2}, \sin \left(\frac{\alpha}{2}\right)\right)} .
$$

Let us now to apply the $n=-1$ approach. The variational method leads to

$$
\lambda \geqslant \frac{K}{\int_{0}^{a} \frac{g(u)}{p(u)} \sin (u) d u},
$$

with

$$
\left(u^{\prime}\right)^{2} g^{\prime}=K
$$

As (22) may be integrated once, we get

$$
p(u)=\sqrt{2 \lambda[\cos (u)-\cos (a)]} .
$$

So, omitting overall multiplicative constants we identify

$$
g^{\prime}(u)=\frac{1}{\cos (u)-\cos (a)},
$$

and integrating, we find for the auxiliary function

$$
g(u)=\frac{1}{\sin (a)} \ln \left[\frac{\sin (a) \tan \left(\frac{u}{2}\right)+1-\cos (a)}{\sin (a) \tan \left(\frac{u}{2}\right)-1+\cos (a)}\right] .
$$

The value of $K$ is found to be

$$
K=2 F^{2}\left(\frac{\pi}{2}, \sin \left(\frac{a}{2}\right)\right)
$$

from (26). Calculating the integral in (25), we obtain

$$
\int_{0}^{a} \frac{g(u)}{p(u)} \sin (u) d u=\frac{2}{\sqrt{\lambda}} F\left(\frac{\pi}{2}, \sin \left(\frac{a}{2}\right)\right),
$$

after some integrations by parts. Finally, from (23), (25), and (29), one gets 


$$
\omega_{n=-1} \leqslant \frac{\pi}{2} \sqrt{\frac{g}{l}} \frac{1}{F\left(\frac{\pi}{2}, \sin \left(\frac{a}{2}\right)\right)},
$$

which coincides within the exact result. If we had made some approximations, as, for instance, take for $g(u)$ or $p(u)$ the same value as for the linear oscillator, the final result had not been equal to the exact one. We show this now for $n=0$. Equation (8) writes in the BD case,

$$
\lambda \geqslant\left(\frac{\pi}{2}\right)^{2}+\frac{4}{\pi a^{3}} \int_{0}^{a} \frac{u N(u, \lambda) d u}{\sqrt{1-u^{2} / a^{2}}}
$$

where we take $N(u, \lambda)=\lambda(u-\sin (u))$. Calculating the integral in (30) and from the definition of $\lambda$, one obtains

$$
\omega_{n=0} \leqslant \sqrt{\frac{g}{l}} \sqrt{\frac{2}{a} J_{1}(a)},
$$

where $J_{1}(a)$ is the Bessel function of the first kind. Because $J_{1}(a)$ alternates the sign, we must restrict the solution given by (31) to those values of $a$ such that $J_{1}(a)$ is positive. For small values of $a$ this condition requires $a \leqslant 3$. Precisely this condition may be recovered from the second variational principle. Taking $f(u)=u-\sin (u)$ and (28) we find from (11) $J_{1}(a) \geqslant 0$. In Fig. 2 we plot the exact solution and the variational solution for the BD's method.

\section{Systems of the form $\ddot{u}+\alpha u^{n}=0$}

We consider now kinds of dynamical systems, which, in their linear approximation $(N=0)$ do not reduce to linear oscillators. These systems may also be treated as the previous ones. First we apply the variational methods and we compare the solution with the exact one. We make, for mathematical simplicity the analysis by using the particular case ${ }^{6} n=3$. It is known that this system describes periodic oscillations. ${ }^{2}$ The system may be integrated once by using the same initial conditions as in previous examples. Hence, we get, after using the independent variable $\tau$,

$$
p(u)=\sqrt{\frac{\alpha \lambda}{2}} \sqrt{a^{4}-u^{4}},
$$

and this one may be integrated once again to get

$$
\omega_{\mathrm{ex}}=\frac{\pi}{2} a \sqrt{\alpha} \frac{1}{F\left(\frac{\pi}{2}, \frac{1}{\sqrt{2}}\right)} .
$$

Applying the first variational method (10) (with $n=-1$ ) one obtains

$$
\alpha \lambda \geqslant \frac{K}{\int_{0}^{a} \frac{u^{3} g(u)}{p(u)} d u}
$$

where $\left(u^{\prime}\right)^{2} g^{\prime}=K$. So, the auxiliary function $g(u)$ is given by

$$
g(u)=\frac{1}{2} \ln \left(\frac{a+u}{a-u}\right)+\arctan \left(\frac{u}{a}\right),
$$

and $K$ by 


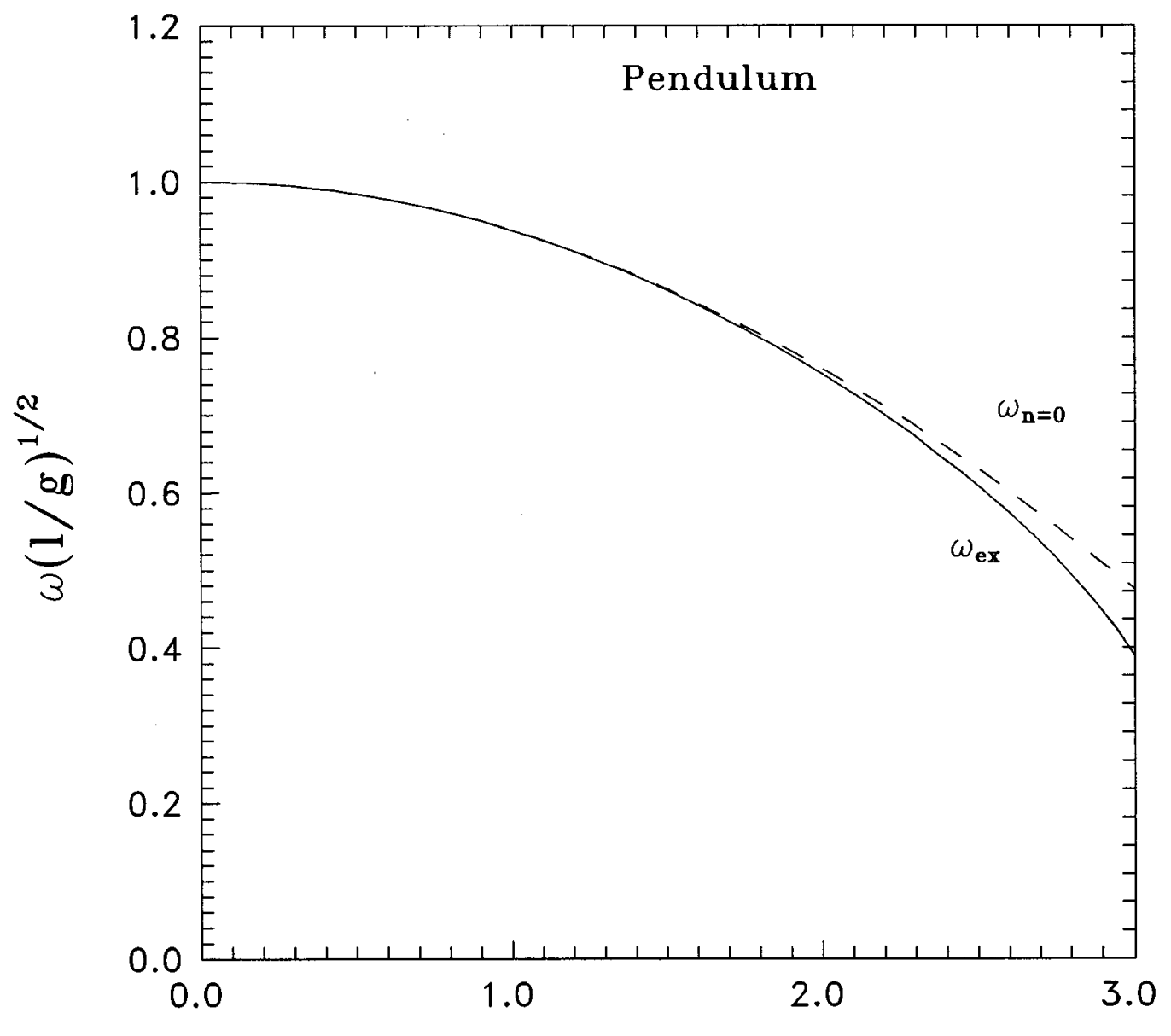

a

FIG. 2. The frequency of the nonlinear pendulum obtained by the BD method ( $\omega_{n=0}$, dashed lines), versus the numerical exact solution $\left(\omega_{\mathrm{ex}}\right.$, solid line).

$$
K(a)=a F^{2}\left(\frac{\pi}{2}, \frac{1}{\sqrt{2}}\right)
$$

The integral involved in (34) is given by

$$
\int_{0}^{a} \frac{u^{3} g(u)}{p(u)} d u=a^{2} \sqrt{\frac{2}{\alpha \lambda}} \int_{0}^{1} \frac{x^{3} d x}{\sqrt{1-x^{4}}}\left[\frac{1}{2} \ln \left(\frac{1+x}{1-x}\right)+\arctan (x)\right]=\frac{a^{2}}{\sqrt{\alpha \lambda}} F\left(\frac{\pi}{2}, \frac{1}{\sqrt{2}}\right) .
$$

Using the previous calculations, we may write (34) in terms of the frequency by

$$
\omega_{n=-1} \leqslant \frac{\pi}{2} a \sqrt{\alpha} \frac{1}{F\left(\frac{\pi}{2}, \frac{1}{\sqrt{2}}\right)}
$$

where the equality gives the exact result (33). On the other hand, we may also apply the BD's method to this case. Taking in (2) $N=\lambda\left(u-u^{3}\right)$ and $n=0$ is (8) we find $K=(\pi / 2)^{3} a^{3}$ and the auxiliary function is given by 


$$
g(u)=\frac{u}{\sqrt{1-u^{2} / a^{2}}} .
$$

We finally find for the frequency

$$
\omega_{n=0} \leqslant \frac{a}{2} \sqrt{3 \alpha}
$$

Calculating numerically $\omega_{n=-1}\left(=\omega_{e x}\right)$ we find $\omega_{\text {ex }}=0.847 a \sqrt{\alpha}$ and $\omega_{n=0} \leqslant 0.866 a \sqrt{\alpha}$. We may observe the good agreement between the exact and the BD's solutions.

\section{DYNAMICAL SYSTEMS DEPENDING ON THE FIRST DERIVATIVE}

We extend here the variational principles found in the first section to nonlinear dynamical systems that contains explicitly the term $\dot{u}$ and powers of it. Some of them are Hamiltonian systems, that is, they have a first integral, and others are dissipative, such as the Van der Pol's equation. We focus our attention to systems of the form

$$
\ddot{u}+\epsilon f(u) \dot{u}^{n}+u=0 .
$$

This equation may be written as

$$
p \frac{d p}{d u}+\lambda u+\epsilon \lambda^{(2-n) / 2} f(u) p^{n}=0 .
$$

By using the change of variables $\tau=2 \omega t / \pi$ and the definition of $\lambda$, we may arrive to

$$
\lambda \geqslant \frac{\epsilon(-1)^{n+1} \lambda^{(2-n) / 2} \int_{0}^{a} f(u) g(u) p^{n} d u+\frac{K}{2}}{\int_{0}^{a} u g(u) d u},
$$

with $\left(u^{\prime}\right)^{3} g^{\prime}(u)=-K$. Notice that in the variational method given by (40) we must know the explicit expression of $p(u)$ and this is only possible if the system admits a first integration. In general, one must use a new approach to (40). In this sense, we derive two new ways to proceed; one of them consists in applying the variational method on the functional of the numerator of (40) but this method only holds if $\int(u)>0$ for any $u \in(0, a)$, the other one consists in making a perturbative expansion on the phase space in order to find an approximate solution for $p(u)$. Let us illustrate the first way.

From (40) we define the functional

$$
J_{g}^{*}[v]=(-1)^{n+1} \int_{0}^{a} f(v) g(v) p^{n} d v=\int_{0}^{1} f(v) g(v)\left(v^{\prime}\right)^{n+1} d \tau,
$$

where $v$ is such that satisfies $v(0)=a, v(1)=0$, and $v^{\prime}<0$ in $\tau \in(0,1)$. Defining $\phi\left(v, v^{\prime}\right)$ $=f(v) g(v)\left(v^{\prime}\right)^{n+1}$, we get from the Euler-Lagrange equations,

$$
n f(\tilde{v}) g(\widetilde{v})\left(\tilde{v}^{\prime}\right)^{n+1}=K^{*}
$$

where $\tilde{v}$ is the value of $v$ such that for a fixed $g, J_{g} \geqslant 0$ has a unique minimum. Notice that $f(\widetilde{v}) \geqslant 0$ is required for any $\widetilde{v} \in(0, a)$, and this is not possible for the Van der Pol's equation. So, once $g$ is determined from $\left(\widetilde{v}^{\prime}\right)^{3} g^{\prime}(\widetilde{v})=-K$, we get

$$
J_{g}[v] \geqslant J_{g}[\tilde{v}]=\frac{K^{*}(a)}{n}
$$

and $K^{*}(a)$ may be calculated from (42). Thus, from (40), we get the variational restriction 


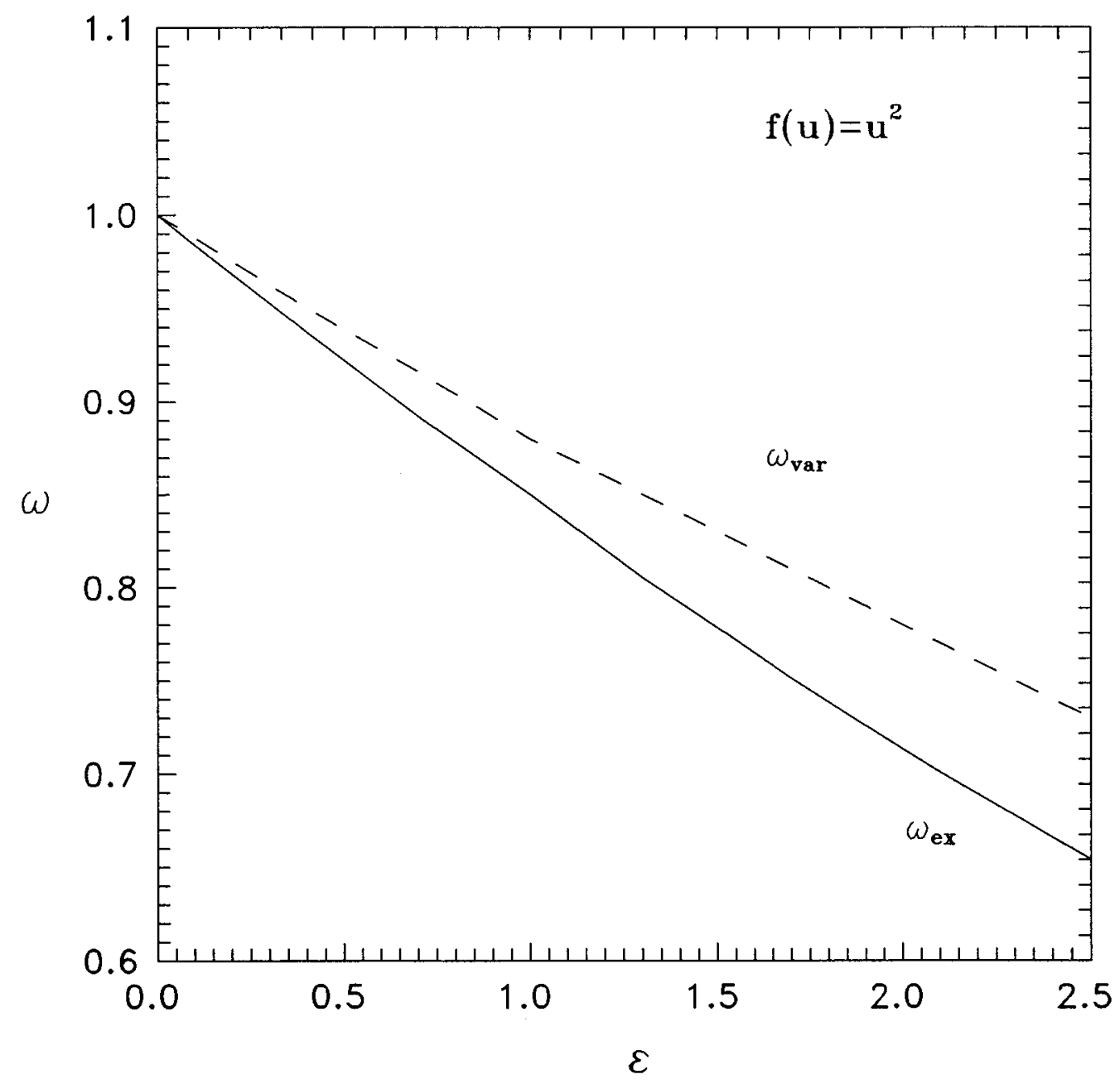

FIG. 3. The frequency of a non-Hamiltonian oscillator given in (46) obtained by the variational method $\left(\omega_{\mathrm{var}}\right.$, dashed lines), versus the numerical exact solution ( $\omega_{\mathrm{ex}}$, solid line).

$$
\lambda \geqslant \frac{\epsilon \lambda^{(2-n) / 2} \frac{K^{*}}{n}+\frac{K}{2}}{\int_{0}^{a} u g(u) d u} .
$$

Taking the auxiliary function $g$ given in the linear case $\epsilon=0$ [Eq. (36)], Eq. (44) becomes

$$
\lambda \geqslant\left(\frac{\pi}{2}\right)^{2}+\frac{4 \epsilon}{\pi a^{3}} \lambda^{(2-n) / 2} \frac{K^{*}}{n} .
$$

Let us to apply this variational method to the specific case

$$
\ddot{u}+\epsilon u^{2} \dot{u}+u=0, \quad \text { with } u(t=0)=a, \quad \dot{u}(t=0)=0 .
$$

In this case, $f(u)=u^{2}$ and $n=1$. From (42) we obtain $K^{*}=\pi^{2} a^{5} / 32$, and from (45),

$$
\omega_{v a r} \leqslant \frac{-b+\sqrt{b^{2}+4}}{2}, \quad \text { with } b=\frac{a^{2} \epsilon}{4} .
$$

In Fig. 3 we plot the exact numerical solution for the frequency versus its variational solution (47). We develop now the second variational method for the specific situation in which $f<0$ for 
some $u \in(a, 0)$. Starting from (40) the problem is to find an expression for $p(u)$. As a first approximation, we may take the expression for $p$ given by the linear case. In this approach we obtain

$$
\lambda \geqslant\left(\frac{\pi}{2}\right)^{2}+\frac{4 \epsilon(-1)^{n+1}}{\pi a^{2}} \lambda \int_{0}^{a} u\left(a^{2}-u^{2}\right)^{(n-1) / 2} f(u) d u .
$$

A better approach may be obtained by the perturbative solution in the phase space. Solving perturbatively (39) for small $\epsilon$, we get

$$
p(u)=p_{0}(u, \lambda)+\epsilon p_{1}(u, \lambda)+O\left(\epsilon^{2}\right),
$$

where

$$
\begin{gathered}
p_{0}(u, \lambda)=\sqrt{\lambda\left(a^{2}-u^{2}\right)} \\
p_{1}(u, \lambda)=-\frac{\lambda^{(5-n) / 2}}{\sqrt{a^{2}-u^{2}}} \int_{a}^{u}\left(a^{2}-z^{2}\right)^{2} f(z) d z .
\end{gathered}
$$

So, the variational method yields

$$
\lambda \geqslant\left(\frac{\pi}{2}\right)^{2}-\frac{4 \epsilon}{\pi a^{2}} \int_{0}^{a} \frac{u f(u)\left(p_{0}+\epsilon p_{1}\right)^{n}}{\sqrt{a^{2}-u^{2}}} d u .
$$

Both approaches are applied as an illustration to the Van der Pol oscillator.

Taking $n=1$ and $f(u)=u^{2}-1$ in (38), we recover the well-known differential for the Van der Pol oscillator,

$$
\ddot{u}+\epsilon\left(u^{2}-1\right) \dot{u}+u=0,
$$

with the initial conditions $u(t=0)=a, \dot{u}(t=0)=0$. It is known that (50) presents a limit cycle for $a=2$. Thus, we study the periodic behavior, and the frequency, in particular, of (50) near of the limit cycle given by $a=2$. In the first approximation (assuming $g$ and $p$ are given by the linear case, $\epsilon=0)$, given by (48) we find for the frequency

$$
\omega_{1} \leqslant \sqrt{1-\frac{2 \epsilon}{\pi}}
$$

Using the second approximation, that is, $p$ given up to first order in $\epsilon$, we obtain

$$
p_{1}(u)=-\sqrt{\lambda} \frac{u}{4}\left(4-u^{2}\right)
$$

and finally

$$
\omega_{2} \leqslant \sqrt{1-\frac{2 \epsilon}{\pi}+\frac{\epsilon^{2}}{4}}
$$

We may solve numerically the Van der Pol's equation for different $\epsilon$ and obtain $\omega_{\text {ex }}$. We plot in Fig. 4 the exact numerical solution and the $\omega_{2}$ solution for the frequency.

\section{CONCLUSIONS}

A variational principle applied to Hamiltonian systems has been developed by Benguria and Depassier. ${ }^{4}$ In this paper we extend their method along the following new lines:

(i) The initial transformation, for Hamiltonian systems, which transforms the differential equation in an integral equation, is generalized by introducing an exponent $n$. The final variational 


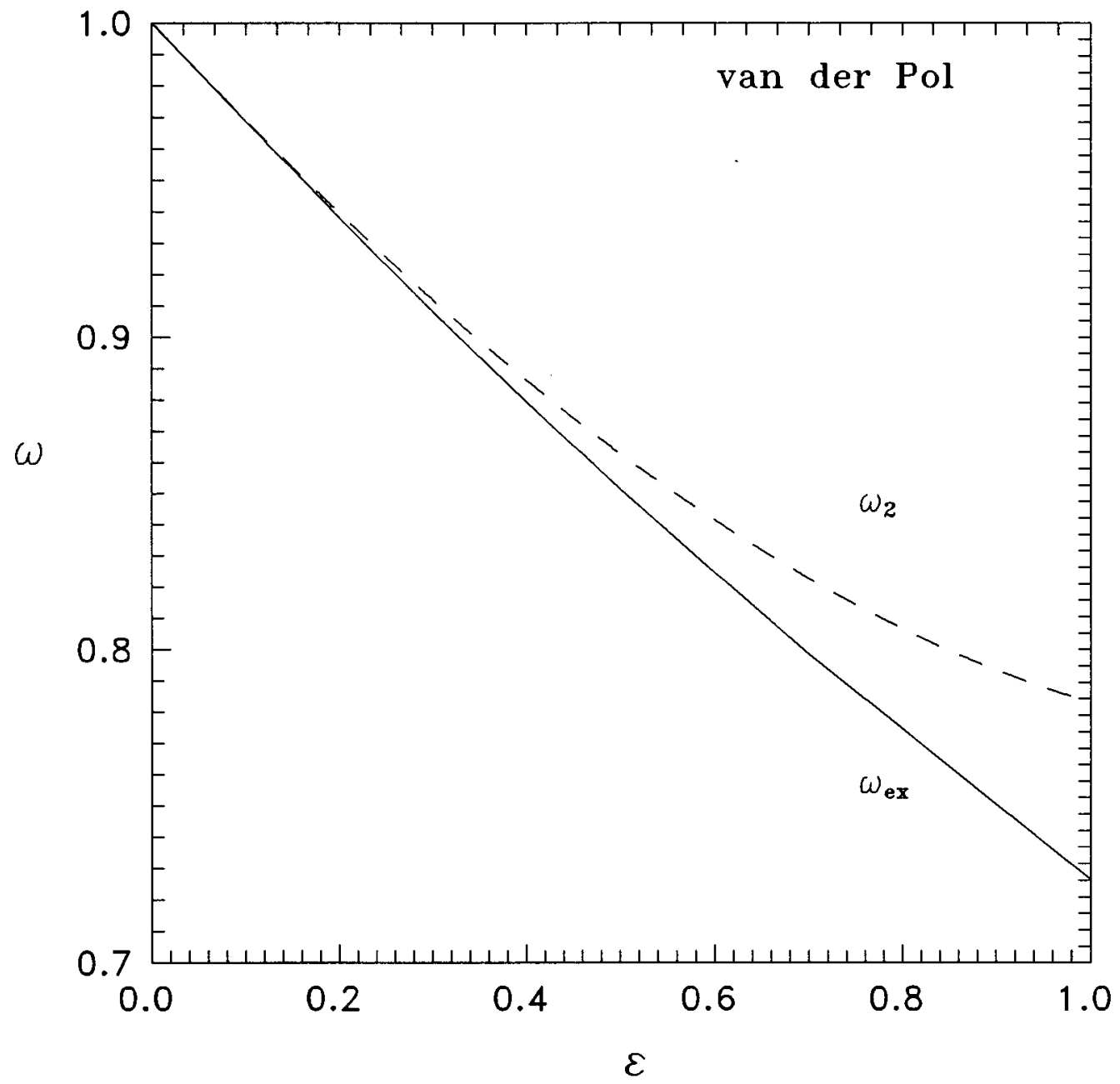

FIG. 4. The frequency of the Van der Pol oscillator obtained by the variational method ( $\omega_{2}$, dashed lines), versus the numerical exact solution $\left(\omega_{\mathrm{ex}}\right.$, solid line).

restriction that relates the frequency with the amplitude, depends strongly on $n$. The variational method proposed by BD only holds for $n>-2$. A second variational method is also applied to Hamiltonian systems and it supplies new constraints between the characteristic parameters involved in the system. We have specified the results to some selected systems as the Duffing oscillator, the nonlinear pendulum, and systems of the form $\ddot{u}+\alpha u^{n}=0$. Better fits those obtained by the method of $\mathrm{BD}$, to the exact solution for the frequency have been discovered.

(ii) The variational principle is also applied to systems with an explicit dependence on the first time derivative. Some of them are Hamiltonian, and they may be exactly characterized. Others are non-Hamiltonian and we may proceed in two different ways. First, we have proposed a new variational method that is applied to two different functionals in order to avoid the explicit dependence on $p(u)$. This method does not hold for systems with $f<0$ for some $u \in(0, a)$. So, we develop an approximate variational method for them. The results are not as good as those obtained for Hamiltonian systems, but they may be understood as upper bounds on the frequency. This approximation consists in finding a perturbative solution for $p$ and the results fit very well for weak nonlinearities.

\section{ACKNOWLEDGMENTS}

I dedicate this paper to Professor José Casas-Vázquez on his 60th birthday. I also would like to thank Professor David Jou for helpful and stimulating discussions. I acknowledge the support of the program Formación de Personal Investigador under Grant No. CLI95-1867 and the Spanish Ministry of Education and Science under Grant No. PB94-0718. 
${ }^{1}$ J. Kevorkian and J. D. Cole, Perturbation Methods in Applied Mathematics (Springer-Verlag, NY, 1981).

${ }^{2}$ R. E. Mickens, An Introduction to Nonlinear Oscillators (Cambridge University Press, Cambridge, 1981).

${ }^{3}$ R. Grimshaw, Nonlinear Ordinary Differential Equations, Applied Mathematical Texts and Engineering Science texts (Blackwell Scientific Publications, Nottingham, Great Britain, 1990).

${ }^{4}$ R. D. Benguria and M. C. Depassier, Phys. Rev. Lett. 77, 2847 (1996).

${ }^{5}$ R. D. Benguria and M. C. Depassier, Phys. Rev. Lett. 77, 1171 (1996).

${ }^{6}$ P. Vainshtein, G. Ziskind, and M. Fichman, Phys. Rev. Lett. 78, 551 (1997). 